\title{
Tungsten as First Wall Material in Fusion Devices
}

\author{
M. Kaufmann, R.Neu \\ Max-Planck-Institut für Plasmaphysik Garching, EURATOM Association, GERMANY
}

\begin{abstract}
The observation in JET of co-deposition of tritium with carbon has led to a broad discussion on the replacement of graphite by a high- $\mathrm{Z}$ material for the first wall coverage. Moreover, due to the high erosion rate, carbon plasma facing components (PFCs) appear to be unacceptable for a commercial fusion reactor. Research in this area has subsequently gained increased attention. This paper describes the status of investigations on the use of tungsten as a First Wall Material. It discusses on the physical side the plasma wall interaction, the transport of tungsten in the plasma boundary and in the core. As an intermediate step on the technological side, graphite is often coated with tungsten layers. For highly loaded surfaces in a fusion reactor finally bulk tungsten components will have to be developed.
\end{abstract}

\section{Keywords}

First wall materials, tungsten, plasma facing components, plasma wall interaction 


\section{Introduction}

Strong cooling of the central plasma has been first observed in the PLT tokamak with a tungsten limiter [1]. Since then graphite has mostly been used in tokamaks as limiter, or in divertor configurations as target plate material. Only a few tokamaks (limiter: FTU [2], TEXTOR [3]; divertor: Alcator C-Mod [4], ASDEX Upgrade [5, 6]) have gained experience with high-Z materials. In machines only partly covered with tungsten a complex migration of carbon from the main chamber into the divertor [7] and the other way around [8] was found. With the observation in JET of strong co-deposition of tritium with carbon [9] together with the results of design studies of fusion reactors [10], it has become clear that in the long run tungsten will be the favoured choice for plasma facing components (PFCs). In particular, the relatively short life time of low-Z material due to erosion is seen as unacceptable in a fusion reactor. Among the high- $Z$ materials, tungsten is the only one with a relatively short activation decay time. Therefore, in the future, JET and ITER will at least partly use tungsten as PFCs.

While for graphite a broad experience is available, tungsten requires intensive research in several areas; specifically in plasma wall-interaction, in the physics of the confined plasma, and in material development. In order to quantify the tungsten influxes and spatially resolve the tungsten concentration a comprehensive spectroscopic diagnostic had to be developed (see for example $[11,12,13]$ ).

In contrast to carbon, the erosion of tungsten by low energy hydrogen atoms is small. However, in the case of tungsten, erosion by fast particles plays an important role. Radiation by carbon in the plasma boundary reduces the load to the target plates and has to be substituted in a pure tungsten machine by artificial impurity gas puffing. Tungsten in the confined plasma is only partly ionized, even at reactor relevant temperatures in the range of 10 to $20 \mathrm{keV}$. The resulting strong radiation thus sets a lower limit for an acceptable tungsten concentration of only a few $10^{-5}$. In connection with the pronounced neoclassical inward drift of high-Z ions, turbulent transport plays a beneficial role here. The inward drift in the pedestal region of an ITER relevant H-mode with type -I ELMs [14] can lead to a further increase.

Technical developments are mostly directed to the specific needs of existing devices where, for example, graphite tiles are often coated with tungsten layers. In future devices like ITER, the solid tungsten armour of the divertor target plates will have to be castellated because of the difference in its thermal expansion compared to the cooling structure.

The plasma wall interaction, discussed in section 2, shows significant differences to carbon - mainly due to the higher atomic mass. Section 3 deals with tungsten transport in the confined plasma covering neoclassical and anomalous effects. A short description of technical questions is given in section 4 . The summary stresses the necessity for further investigations. 


\section{Plasma Wall Interaction with Tungsten}

One of the main advantages of tungsten is its low permanent hydrogen retention $[15,16]$ at elevated temperatures. H-retention by co-deposition does not play a significant role with tungsten due to the low expected erosion rates and the absence of a process similar to that of hydro-carbon formation. The implications of the formation of $\mathrm{H}$ bubbles, as well as of the blistering due to He, observed in laboratory experiments after irradiation with high fluencies of $\mathrm{H}$ or He respectively, are still under discussion (see for example [17]).

Because of the higher atomic mass substantial sputtering by deuterium occurs only at relatively high energies (e.g. sputtering rate $10^{-3}$ for $400 \mathrm{eV}$ ions) so that sufficiently cold deuterium plasma in the SOL and in front of the target plates does not lead to substantial erosion. In addition, because of their low speed, W-neutrals sputtered from the wall are ionized over distances small compared to their large gyro radius. This leads to a prompt redeposition of a high fraction of sputtered particles [18].

In tokamaks with graphite surfaces, carbon plays an important role as a radiator in the plasma boundary leading to a substantial reduced heat load on the target plates. With a first wall of a high Z-material, the carbon radiator has to be replaced by Argon or Neon for example $[19,20]$. While with graphite surfaces, a degree of self regulation can be observed, the addition of a noble gas has to be carefully controlled. To this end, the measurement of currents through the target plates induced by thermal forces seems to be a robust method for the control of the divertor electron temperature [19]. While the erosion of tungsten surfaces by low temperature hydrogen can usually be neglected this is not the case for these admixtures if the divertor plasma temperature is too high [11, 21, 22]. In a limiter configuration, tungsten erosion is in any case unacceptably high while with a divertor the plasma temperature in front of the target plates has to stay below about $5 \mathrm{eV}$ [23]. It requires in the divertor configuration a strong reduction of the energy transport during ELMs compared to that obtained with the standard type-I-ELMs. This can be achieved by external means e.g. an increase in the ELM frequency [24] or by increasing the overall edge transport with edge resonant magnetic field perturbations [25]. A specific effect in the main chamber can be observed during ELMs. While the main fraction of the ELM energy loss goes into the divertor, filaments with a helical structure [26] transport plasma to the wall of the main chamber. Impurities in this typically $\mathrm{T}_{\mathrm{i}} \approx 50 \mathrm{eV}$ plasma [27] have the potential to sputter tungsten. Erosion of the main chamber by CX-particles may also lead to substantial tungsten fluxes if, in some confinement scenarios, the plasma edge temperature is relatively high [20].

Another source for erosion are fast particles from additional heating. Fast deuterium ions generated by Neutral Beam Injection can be lost due to drifts or MHD-effects, like ELMs. Their effect on tungsten erosion of protection limiters has been observed with high time resolution [28, 29]. In contrast, for the case of ICRH, tungsten erosion is not dominated by fast particles [30, 28, 31]. Local observation on limiters shows tungsten influxes created immediately after the ICRH is switched on, which suggests that an increase of the sheath potential in the neighbourhood of the antenna is responsible. However, variations of the field line angle with respect to the Faraday screen - which should lead to a strong variation of the electric potential - do not show a clear effect. The limited theoretical understanding 
poses a challenge for the combination of ICRH and a tungsten covered wall. The erosion of tungsten by ICRH (and of course by other sources) can strongly be reduced by a boronization of the surfaces, but boron disappears after a few discharges on heavy loaded areas [32, 30, 33].

\section{Edge and Core Transport}

In the region of open field lines, the transport of tungsten has been described by the DIVIMP-code based on a B2-EIRENE plasma background [34]. These results have been extrapolated to ITER [35].

In the H-mode, one has to subdivide the confined plasma into the pedestal and core regions. In the reactor relevant regime of type-I ELMs, the pedestal, with its steep pressure gradient, breaks down during an ELM and a substantial part of the pedestal plasma is ejected. In between ELMs, tungsten moves into the pedestal region due to strong inward particle drift [14]. If the next ELM comes in due time then this tungsten is removed. One can clearly observe this effect as a function of the ELM frequency (see figure 1) [24]. This suggests that ELMs are a necessary prerequisite for sufficiently low tungsten content in the plasma. For ITER or DEMO this demand is in line with the necessity to keep the energy per ELM small (see above).

In the core plasma, however, an inward particle drift can lead to accumulation in the centre. In the simple picture, neoclassical theory predicts a diffusion coefficient which scales proportional to $1 / Z^{2}$ while the inward drift is proportional to $1 / Z$. If the deuterium density profile is not particularly flat this, in general, leads to a strong accumulation of tungsten in the core. The neoclassical accumulation has been experimentally observed in several occasions [36, 6, 37, 38, 39]. However, if the heat flow in the core is sufficiently high then anomalous transport can easily exceed the neoclassical effects, especially of high- $Z$ ions because of the $1 / Z$ and $1 / Z^{2}$ scaling respectively. One can experimentally demonstrate this effect by, for example localized central ECRH which stimulates the anomalous turbulent transport $[40,6,41]$ as demonstrated in figure 2. There, the top three rows depict the trajectories of the additional heating (please note the small contribution of central ECRH) and the temporal behaviour of the $\mathrm{H}$ factor, the $\mathrm{H}_{\alpha}$ light and the electron temperature at two radial positions. The tungsten content drops with central heating and recovers without, which can be deduced from the comparison of central and edge $\mathrm{W}$ concentration $\left(\mathrm{C}_{\mathrm{W}}\right)$ shown in the lowest row. There are two ways the anomalous transport can influence the Wprofile. On the one hand it can overcome the Ware-pinch of the main ions, thus flattening the deuterium density profile (second lowest row in fig.2), while on the other hand anomalous transport can directly determine the tungsten flux. The discharge in figure 2 does not develop sawtooth activity, which in ordinary H-Mode discharges also expells the central impurity content (see for example [14]). Recent theoretical work [43] shows two dominant turbulent transport mechanisms for high Z-ions. Neither of them leads to a substantial accumulation of tungsten with respect to the deuterium density. In summary, this leads to the expectation that peaked $\mathrm{W}$ concentration profiles are unlikely to exist in the type-I-ELMy H-Mode of a burning device. Combined with a high density, low temperature edge plasma, an all tungsten device seems to be feasible [20], although the details of the radial transport still have to be quantified. 


\section{Technological Developments}

On the technical side, the critical issues are the mechanical properties of tungsten. Although its strength is high, its tensile elongation at room temperature is almost zero, making it brittle. The ductile to brittle transition temperature (DBTT) is far above room temperature, depending on the details of manufacture. In order to improve the brittleness several kinds of tungsten based alloys have been developed.

In most present day devices, inertial cooling is sufficient due to the short pulse durations. The low particle fluencies, and therefore, the limited erosion, enable the use of $\mathrm{W}$ coatings on a graphite substrate. In steady state devices, actively cooled components will be necessary and for high heat flux components, the bonding between the $\mathrm{W}$ armour and the heat sink is of special concern. In the case of ITER, copper alloys are proposed as the heat sink material. Since copper exhibits a much larger thermal expansion than tungsten, castellated or 'brush'-like structures will have to be used. In order to operate at higher cooling medium temperatures, concepts of actively cooled tungsten structures are under development for DEMO.

Specific developments are aimed at suppressing the production of volatile tungsten oxide in case of a loss-of-coolant accident with air ingress so as to reduce the risk of releasing radioactive material. The admixture of small amounts of silicon and chromium to tungsten leads to a glassy protection layer on the tungsten surface, which can reduce the tungsten oxidation rate by up to four orders of magnitude (see Fig. 3) [44].

\subsection{Tungsten Coatings}

Graphite or carbon-fibre composites (CFC) have a reduced electrical conductivity compared to metals which helps to keep eddy and halo currents low. In contrast, tungsten has a conductivity 200 times as large as carbon based materials and its mass density is larger by a factor of 8.5. These properties would lead to a considerable higher load on the support structures if bulk tungsten tiles were used making the transition from a device designed for C-based PFCs very costly and time consuming. In principle, castellation of the W components could be used to reduce eddy currents, but the technical effort is quite high (see for example [45]). Therefore, several present day devices have chosen the coating solution, when implementing tungsten PFCs. A variety of techniques are available, amongst which physical vapour deposition (PVD) [46, 47, 48] and plasma spray (PS) [46, 49] are most commonly used for fusion applications to produce thin ( $\mu \mathrm{m}$ scale) or thick (mm scale) coatings, respectively. They can withstand heat loads in excess of $10 \mathrm{MW} / \mathrm{m}^{2}$ with typical erosion rates below $1 \mathrm{~nm} / \mathrm{s}$ [50] and they can survive $10^{4}-10^{9}$ seconds of plasma operation. Chemical vapour deposition (CVD) has been investigated as a method for in-situ W-coating of PFCs in fusion devices [51]. Recently, JET has launched a test program in the framework of the 'ITER-like wall project' to investigate PVD, CVD as well as PS coatings on CFC substrate. Although the thermal expansion of CFC is strongly anisotropic and does not match that of tungsten, PVD and PS coatings could be identified in thermal screening as well as cyclic loading experiments to serve the JET requirements [52]. 
In future fusion devices, a few mm of tungsten armour on the main chamber first wall might be sufficient and W-PS coatings on low activation steel may be an attractive solution $[53,54]$.

\subsection{Bulk Tungsten Components}

In the divertor of ITER or a reactor, bulk tungsten solutions have to be foreseen. TEXTOR has performed experiments with bulk $\mathrm{W}$ limiters to explore their behaviour under fusion relevant particle and power loads. Particularly, the influence and performance of castellated structures were investigated [55]. JET plans to address the issue of melt layer erosion within the 'ITER-like wall project' with a W lamellae design, which was successfully tested by cyclic loading at $\approx 8 \mathrm{MW} / \mathrm{m}^{2}$ [45]. The components in ITER will be actively cooled and the cooling structure will be made from CuCrZr. An efficient solution to overcome the different thermal expansion of the $\mathrm{Cu}$ alloy and $\mathrm{W}$ is the use of castellated structures or so called 'macro-brushes' [56] or 'mono-blocks' [57]. Such components have already been tested with power loads up to $25 \mathrm{MW} / \mathrm{m}^{2}$ for 1000 cycles, thereby accomplishing the ITER design values [57].

In a reactor, the technical concept will depend on the cooling medium, e.g. water or helium. The latter requires relative high pressures but allows high coolant temperatures. In such a case, the cooling structure itself could be designed from $\mathrm{W}$ components. Fig. 4 shows the concept of ' $W$-thimbles' which are designed as part of a He cooled W divertor in DEMO [58].

\section{Summary}

In a fusion reactor, graphite as a first wall material will, with a very high probability, have to be replaced by tungsten. So far, plasma experiments have demonstrated that in most scenarios the tungsten erosion of the surfaces and its concentration in the central plasma can be kept sufficiently low. In certain scenarios with high edge temperatures this may, however, not be the case. In addition, the high erosion in the neighbourhood of an ICRH antenna needs particular attention. As an intermediate solution, the coating of graphite with tungsten is an available technology. Technological solutions for the highly loaded divertor targets in a fusion reactor are under development. The relatively high ductile to brittle transition temperature, however, poses specific problems. Altogether tungsten as the first wall material looks promising, but several open questions still remain to be solved.

\section{Acknowledgement}

The authors would like to thank H. Bolt, R. Dux and A. Kallenbach for helpful discussions. 


\section{References}

[1] V. Arunasalam et al., 'Recent Results from the PLT Tokamak' in Proc. 8th Conf. EPS, Prague 1977, EPS, Geneva, 1978, vol.2, p.17

[2] M.L. Apicella et al., Nucl. Fusion 37 (1997) 381

[3] A. Pospieszczyk et al., J. Nucl. Mater. 290-293 (2001) 947

[4] B. Lipschultz et al., Nucl. Fusion 41 (2001) 585

[5] R. Neu et al., Plasma Phys. Control. Fusion 38 (1996) A165

[6] R. Neu et al., Nucl. Fusion 45 (2005) 2009

[7] H. Maier et al., J. Nucl. Mater. 266-269 (1999) 1003

[8] A. Kallenbach et al., 'Spectroscopic investigation of carbon migration with tungsten walls in ASDEX Upgrade', to be published in J. Nucl. Mater.

[9] P. Coad et al., J. Nucl. Mater. 290-293 (2001) 224

[10] H. Bolt et al., J. Nucl. Mater. 307-311 (2002) 43

[11] A. Thoma et al., Plasma Phys. Control. Fusion 39 (1997) 1487

[12] A. Pospieszczyk et al., to be published

[13] K. Asmussen et al., Nucl. Fusion 38 (1998) 967

[14] R. Dux et al., Fusion Technol. 44, (2003) 708

[15] R. Causey, J. Nucl. Mater. 300 (2002) 91

[16] J. Roth, M. Mayer, J. Nucl. Mater. 313-316 (2003) 469

[17] D. Nishijima et al., Nucl. Fusion 45 (2005) 669

[18] D. Naujoks et al., Nucl. Fusion 36 (1996) 671

[19] A. Kallenbach et al., J. Nucl. Mater. 337-339 (2005) 732

[20] A. Kallenbach et al., Plasma Phys. Control. Fusion 47 (2005) B207

[21] K. Krieger, H. Maier, R. Neu, ASDEX Upgrade team, J. Nucl. Mater. 266-269 (1999) 207

[22] B. Unterberg et al., J. Nucl. Mater. 266-269 (1999) 75]

[23] G. Federici et al., Plasma Phys. Control. Fusion 45 (2003) 1523-1547

[24] P. Lang et al., Nucl. Fusion 45 (2005) 502

[25] T. Evans et al., Nucl. Fusion 45 (2005) 595

[26] A. Kirk et al., Phys. Rev. Let., 96 (2006) 185001

[27] A. Herrmann et al., 'The filamentary structure of ELMs in the scrape-off layer in ASDEX Upgrade', accepted for publ. in J. Nucl. Mater.

[28] R. Dux et al., 'Tungsten at the ICRH limiters in ASDEX Upgrade', accepted for publ. in J. Nucl. Mater.

[29] M.Y.Ye et al, J. Nucl. Mat. 337-339 (2005) 104

[30] B. Lipschultz et al., Phys. Plasmas 13 (2006) 056117

[31] R. Dux et al., J. Nucl. Mater. 337-339 (2005) 852

[32] M.L. Apicella, et al., J. Nucl. Mater. 313-316 (2003) 269

[33] Vl.V. Bobkov et al., 'Compatibility of ICRF antennas with W-coated limiters for different plasma geometries in ASDEX Upgrade', accepted for publ. in J. Nucl. Mater.

[34] A. Geier et al., J. Nucl. Mater. 313-316 (2003) 1216 
[35] K. Schmid, K. Krieger, A. Kukushkin, A. Loarte, 'DIVIMP modelling of tungsten impurity transport ITER', accepted for publ. in J. Nucl. Mater.

[36] J. Rapp et al., Plasma Phys. Control. Fusion 39 (1997) 1615

[37] R. Dux, C. Giraud, K.D. Zastrow, JET EFDA contrib., Nucl. Fusion 44 (2004) 260

[38] D. Pacella et al., Phys. Rev. E. 61 (2000) 5701

[39] J. Rice et al., Nucl. Fusion 42 (2002) 510

[40] R. Neu et al., Plasma Phys. Control. Fusion 44 (2002) 811

[41] W.P. West et al., Phys. Plasma 9 (2002) 1970

[42] R. Dux et al., J. Nucl. Mat. 313-316 (2003) 1150-1155

[43] C. Angioni, A.G. Peeters, Phys. Rev. Let. 96 (2006) 095003

[44] F. Koch, H. Bolt, 'Self Passivating W-based Alloys as Plasma Facing Material for Nuclear Fusion’, Physica Scripta T128 (2007) 100

[45] T. Hirai et al., 'R\&D on full tungsten divertor and beryllium wall for JET ITER-like wall project', this conference

[46] C. Garcia-Rosales et al., Fusion Technol. 32 (1997) 263

[47] H. Maier, Materials Science Forum 475-479 (2005) 1377

[48] S. Lehto et al., Fus. Eng. Design 66-68 (2003) 241

[49] X. Liu et al., J. Nucl. Mater. 329-333 (2004) 687

[50] M. Mayer et al., 'Tungsten erosion in the outer divertor of JET', accepted for publ. in J. Nucl. Mater.

[51] A. Cambe, E. Gauthier, J.M. Layet, S. Bentivegna, Fus. Eng. Des. $56-57$ (2001) 33

[52] H. Maier et al., 'Tungsten coatings for the ITER-like wall project', accepted for publ. in J. Nucl. Mater.

[53] H. Greuner et al., Fus. Eng. Design 75-79 (2005) 333

[54] N.P. Taylor, R. Pampin, Eng. Design 81 (2006) 1333

[55] T. Hirai et al., J. Nucl. Mater. 313-316 (2003) 67

[56] J.W. Davis et al., J. Nucl. Mater. 258-263 (1998) 308

[57] J. Linke, Physica Scripta T123 (2006) 45

[58] W. Krauss, N. Holstein, J. Konys, Fus. Eng. Design 75-79 (2005) 775 


\section{Figure Captions}

Fig. 1: ELMs counteract to the inward drift in the pedestal region: an increase of the ELM frequency reduces the W-concentration [24].

Fig. 2: Central heating with ECRH und the related turbulence suppresses central Waccumulation [42] (for details please see text).

Fig. 3: The oxidation of tungsten is suppressed by admixtures of silicon and chromium [44].

Fig. 4: Principle layout of $\mathrm{W}$ cooling tubes and different array structures for a He cooled divertor [58]. 
Figures

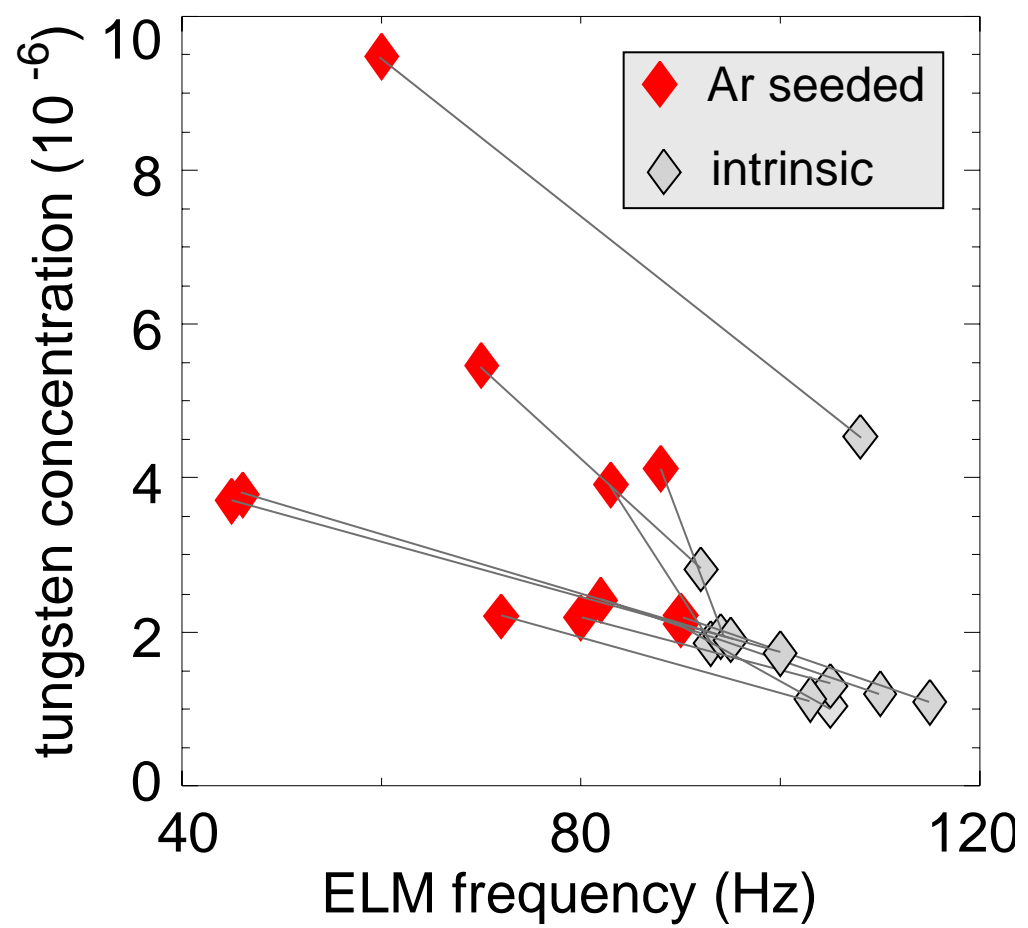

Fig. 1: ELMs counteract to the inward drift in the pedestal region: an increase of the ELM frequency reduces the $\mathrm{W}$-concentration [24]. 


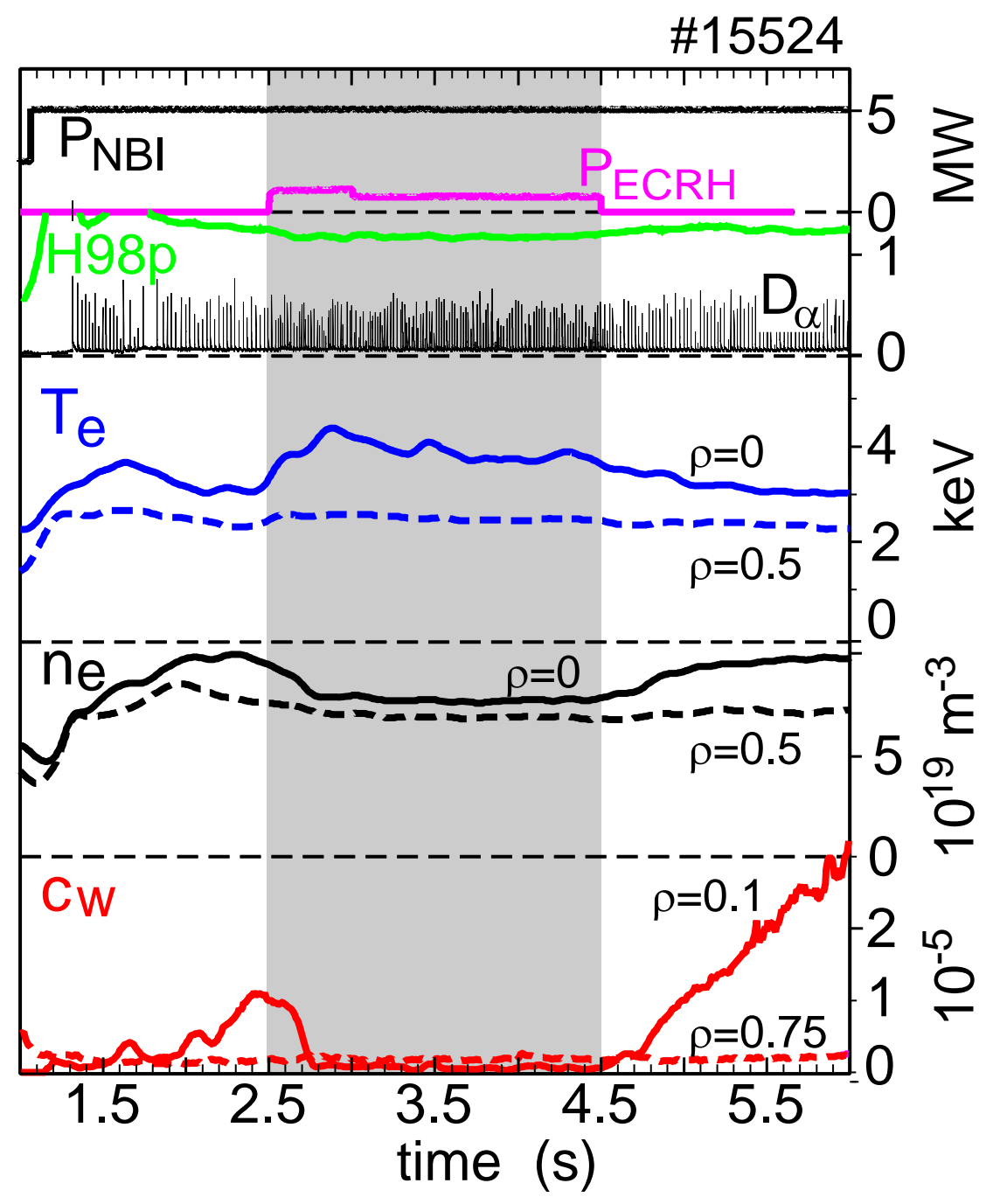

Fig. 2: Central heating with ECRH and the related turbulence suppresses central Waccumulation [42] (for details please see text). 


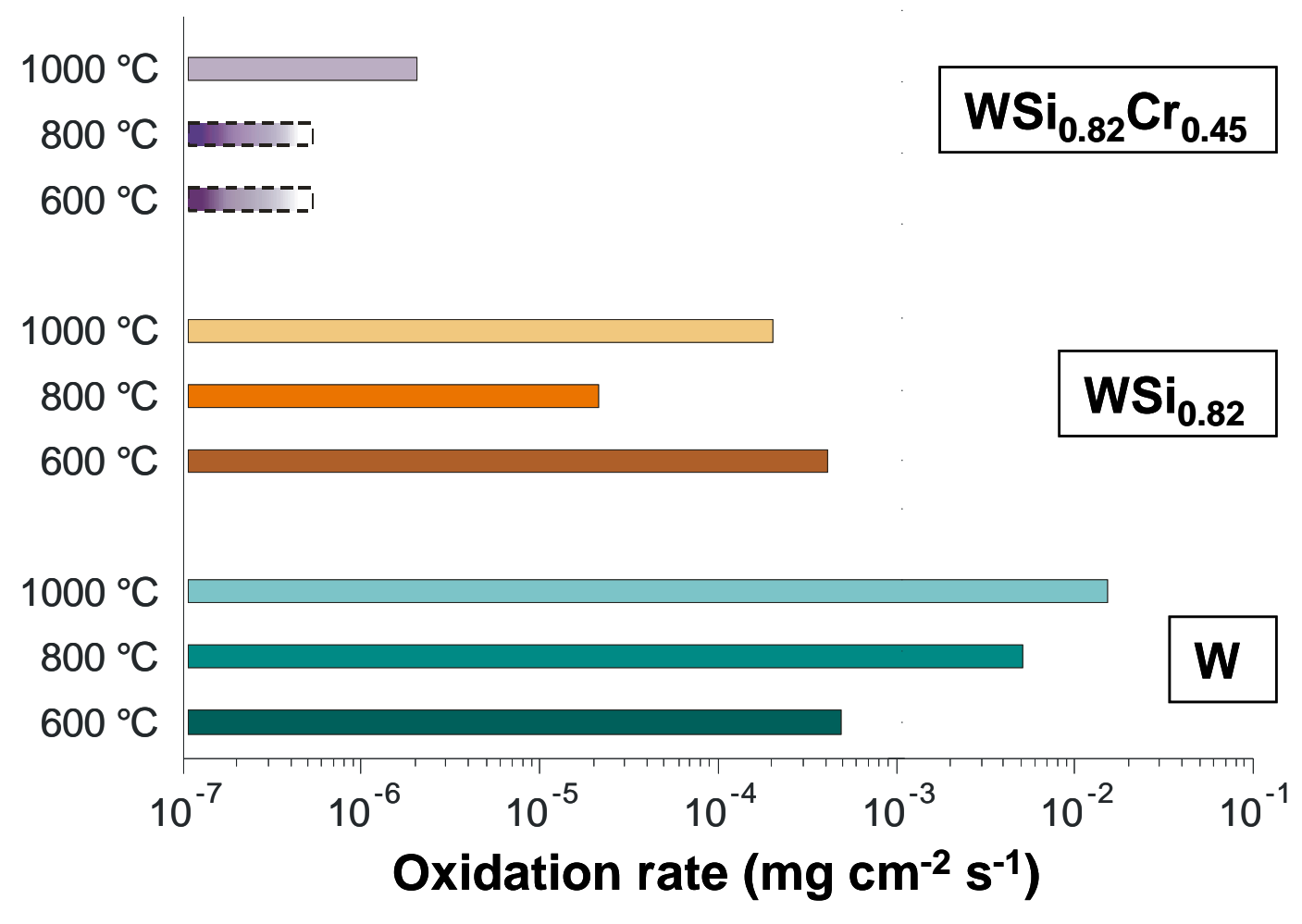

Fig. 3: The oxidation of tungsten is suppressed by admixtures of silicon and chromium [44]. 


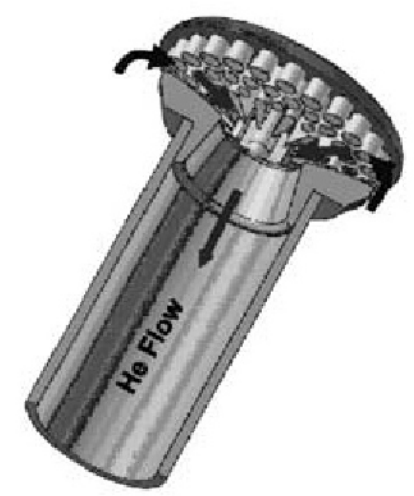

He flow in pin array and support tube

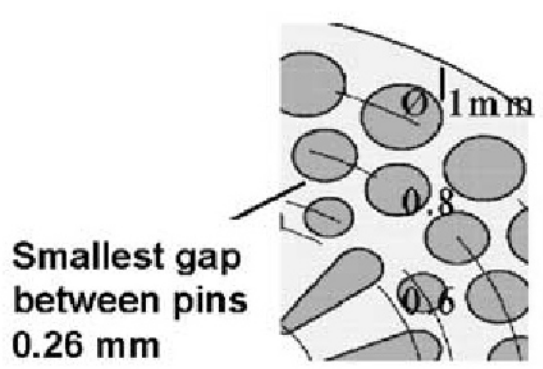

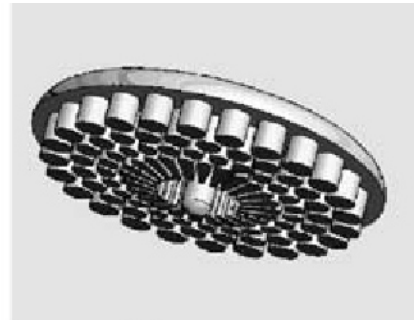

Pin array

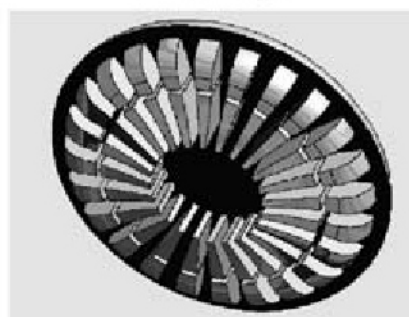

Straight slot array

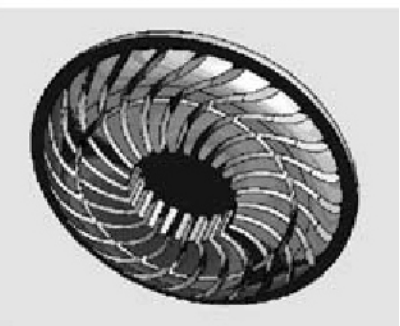

Curved slot array

Fig. 4: Principle layout of $\mathrm{W}$ cooling tubes and different array structures for a He cooled divertor [58]. 